\title{
DESENVOLVENDO COMPETÊNCIAS NO LABORATÓRIO DE FÍSICA EM CURSOS DE ENGENHARIA
}

\author{
DEVELOPING SKILLS IN THE PHYSICS LAB IN ENGINEERING COURSES
}

Eliane Fátima Chinaglia ${ }^{1}$, Sueli Hatsumi Masunaga ${ }^{2}$, Simone Camargo Trippe ${ }^{3}$

DOI: 10.37702/REE2236-0158.v40p160-172.2021

\begin{abstract}
RESUMO
Neste trabalho, apresentamos um procedimento experimental e uma análise de dados como base para o desenvolvimento de competências propostas no novo plano pedagógico dos cursos de engenharia. O envolvimento do aluno na configuração do experimento, na aquisição e processamento de dados, na identificação de fontes de erros experimentais e na validação de uma aproximação teórica pode estimular o pensamento crítico e a reflexão sobre os resultados obtidos. Dessa forma, espera-se incentivar o desenvolvimento de competências associadas à $(i)$ aplicação de métodos científicos para a análise de fenômenos físicos, (ii) condução de experimentos e interpretação de resultados e (iii) modelagem matemática de sistemas físicos. Propomos a aplicação de uma atividade, "O experimento do pêndulo simples", com o objetivo de promover o desenvolvimento dessas competências requeridas do engenheiro atual, explicitando as habilidades que os estudantes desenvolverão em cada etapa. Essa atividade pode ser adaptada no cenário atual da virtualização das aulas devido à pandemia da COVID19 e em modalidade de educação a distância.
\end{abstract}

Palavras-chave: desenvolvimento de competências; pêndulo simples; modelos teóricos; oscilações amortecidas; videoanálise.

\begin{abstract}
In this work, a laboratory study was conducted, and an analysis procedure was applied as a basis for the development of skills proposed in the new pedagogical plan for engineering courses. The student's engagement in the configuration of the experiment, in data acquisition and processing, in identifying sources of experimental errors and in validating a theoretical approach may stimulate the development of critical thinking and a reflection on the obtained results. Thus, this procedure is expected to encourage the development of skills associated with ( $i$ ) applying scientific methods for the analysis of physical phenomena, (ii) conducting experiments and interpreting results, and (iii) employing mathematical modeling of physical systems. We propose the application of an activity, "The simple pendulum experiment", aimed at promoting the development of fundamental skills required of current engineers, underlining which skills the students could be developing at each stage. This activity can be adapted to the current process of virtualization of classrooms due to the COVID19 pandemic and to the distance learning programs.
\end{abstract}

Keywords: Skills development; Simple pendulum; Theoretical models; Damping; Video analysis.

1 Profa. Dra. em Ciências, Centro Universitário FEI, São Bernardo do Campo - SP, echinaglia@ fei.edu.br 


\section{INTRODUÇÃO}

A discussão sobre formação em engenharia baseada em competências tem sido ampliada e intensificada nos últimos anos. Dessa forma, o conteúdo disciplinar passa a ser um instrumento para o desenvolvimento de competências e não o objetivo final, per se, a ser alcançado. $\mathrm{O}$ desenvolvimento de habilidades como solução de problemas complexos e mal estruturados, criatividade, pensamento crítico, liderança, planejamento e aprendizagem de forma autônoma tem um papel fundamental na formação do engenheiro atual (ABENGE, 2018; CNI, 2020; RUGARCIA, 2000; WANKAT; BULLARD, 2016; WORLD ECONOMIC FORUM, 2018).

No Brasil, a implementação do aprendizado por competências vem sendo realizada em várias instituições (ABENGE, 2018; CNI, 2018; CNI, 2020; MEC, 2019), tornando necessárias, como parte dessa implementação, a elaboração de atividades e a disponibilidade de ambientes e condições que incentivem o desenvolvimento das habilidades e conteúdos desejados. Nesse sentido, a aplicação de atividades que permitam que os alunos trabalhem seu pensamento crítico em trabalhos experimentais, em desenvolvimento de projetos e em resolução de problemas é fundamental para o seu desenvolvimento (WANKAT; BULLARD, 2016).

A atividade que propomos para esse desenvolvimento é a utilização de um experimento de baixo custo e de fácil montagem, mas que apresente alguns desafios experimentais. Assim, escolhemos o experimento do pêndulo simples que é comumente utilizado nos cursos de graduação na área de exatas por apresentar vantagens tanto no aspecto teórico quanto no experimental. Por ser simples, esse experimento pode ser facilmente montado e modificado, inclusive, em casa pelos estudantes, podendo proporcionar o desenvolvimento das competências mesmo com a virtualização das aulas em meio à pandemia da COVID-19 ou na modalidade de educação a distância.

Ao trabalhar o desenvolvimento de competências na condução de um experimento, os dados e as análises são revisitados ou refeitos após as considerações dos limites de aplicação de um modelo e das condições experimentais. No caso do experimento do pêndulo simples, quando os dados são adquiridos com o devido cuidado em relação aos parâmetros experimentais, sua análise resulta em valores acurados da aceleração da gravidade. Esse resultado, portanto, pode ser atribuído ao aprendizado adquirido no processo de montagem do experimento e na aquisição e análise dos dados, como consequência do desenvolvimento dessas competências.

Além dos desafios experimentais, os conceitos associados com a força restauradora, o movimento harmônico simples e o oscilador harmônico amortecido podem ser abordados do ponto de vista teórico, e uma rica discussão sobre aproximações e modelos matemáticos pode ser realizada.

Para desenvolver competências associadas à (i) aplicação de métodos científicos para a análise de fenômenos físicos, (ii) condução de experimentos e interpretação de resultados e (iii) modelagem matemática de sistemas físicos (CNI, 2018; CNI, 2020), é importante que o aluno se envolva com o experimento, desenvolvendo a habilidade de configurá-lo de forma adequada, pensando na aquisição e processamento de dados, identificando fontes de erros experimentais e validando uma aproximação teórica. Adicionalmente aos aspectos mencionados acima, o pensamento crítico pode ser estimulado, levando o estudante a refletir sobre um resultado inicial obtido e avaliar a necessidade de correções e conjeturar sobre os parâmetros ou modelos teóricos ou ainda procedimentos experimentais que podem estar afetando o resultado.

O desenvolvimento dessas habilidades pode ser realizado no experimento de pêndulo simples, levando em consideração tanto o amortecimento do sistema quanto o fato do período do pêndulo simples ser válido para aproximações de pequenos ângulos. Para amplitudes angulares relativamente maiores, o movimento deve ser descrito por uma equação diferencial não linear, cuja solução exata é apenas obtida numericamente para uma dada condição inicial. Dessa forma, surgem vários 
modelos que descrevem o período do sistema levando em consideração a amplitude angular. Entretanto, esses modelos também são aproximações, com algum erro percentual em relação à solução exata, o que oferece oportunidades para uma discussão mais aprofundada em sala de aula.

Assim, o objetivo deste artigo é propor e pontuar os desafios da construção de um procedimento experimental para aquisição e análise de dados do movimento de um pêndulo simples, considerando tanto o amortecimento do sistema quanto a amplitude inicial do lançamento. A partir dos dados obtidos, os alunos podem avaliar os limites de validade de um modelo teórico, além de analisar quais são as condições experimentais adequadas que levam à determinação acurada da aceleração da gravidade local. Como consequência de todos esses desafios experimentais e análise dos limites de aplicabilidade de modelos teóricos, objetivando o desenvolvimento das competências desejadas, o aluno chegará a valores aceitáveis da aceleração da gravidade local, podendo, assim, avaliar criticamente seu procedimento ao longo da experiência.

\section{Pêndulo Simples}

Em um pêndulo simples, um corpo de massa $m$ acoplado a um fio ideal e inextensível de comprimento $L$ realiza um movimento periódico de período $T$ quando retirado de um ângulo inicial $\theta_{o}$ da sua posição de equilíbrio. A Figura 1 apresenta o diagrama de forças que atuam sobre o corpo em um sistema sem amortecimento. A análise do movimento desse corpo pode ser realizada resolvendo-se a equação homogênea de $2^{\mathrm{a}}$ ordem:

$$
\frac{d^{2} \theta}{d t^{2}}+\frac{g}{L} \operatorname{sen} \theta=0
$$

onde $g$ é a aceleração da gravidade local e $\theta$ o ângulo de oscilação. A solução exata da equação (1) para o período de oscilação é a integral elíptica cuja solução pode ser encontrada numericamente (MARION; THORNTON, 1988):

$$
T=4 \sqrt{\frac{L}{g}} \int_{0}^{\frac{\pi}{2}} \frac{d \theta}{\sqrt{1-\operatorname{sen}^{2}\left(\frac{\theta_{o}}{2}\right) \cdot \operatorname{sen}^{2}(\theta)}} .
$$

Vários modelos que consideram a amplitude angular no cálculo do período podem ser encontrados na literatura, com maior ou menor grau de desenvolvimento. Com o intuito de que os estudantes desenvolvam competências associadas a modelamentos matemáticos de dados experimentais, três modelos propostos na literatura foram escolhidos para a análise dos dados. Aqui, as expressões desses modelos são apresentadas abaixo apenas na sua forma final:

Expressão 1 - série infinita até o $2^{\circ}$ termo (FULCHER; DAVIS, 1976):

$$
T_{1}=2 \pi \sqrt{\frac{L}{g}} \cdot\left[1+\frac{1^{2}}{2^{2}} \operatorname{sen}^{2}\left(\frac{\theta}{2}\right)\right]
$$

Expressão 2 - Bernoulli (SMITH, 1960):

$$
T_{2}=2 \pi \sqrt{\frac{L}{g}} \cdot\left(1+\frac{1}{16} \theta^{2}\right)
$$

Expressão 3 - Lima-Arun (2006):

$$
T_{3}=-2 \pi \sqrt{\frac{L}{g}} \cdot\left\{\frac{\ln \left[\cos \left(\frac{\theta}{2}\right)\right]}{1-\cos \left(\frac{\theta}{2}\right)}\right\}
$$

Figura 1 - Diagrama de forças para um pêndulo de massa $m$ e comprimento $L$

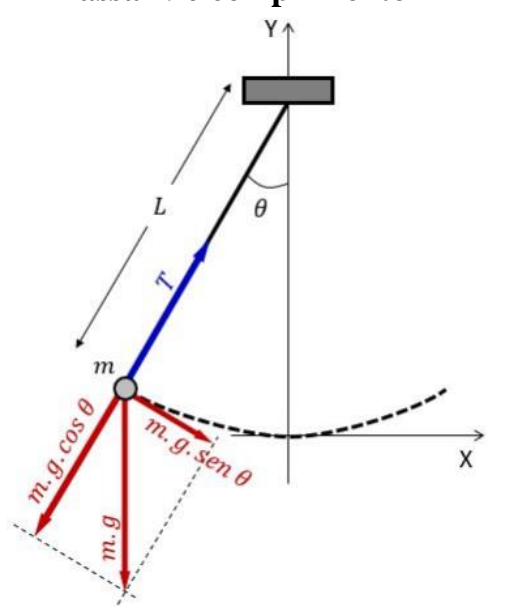

Fonte: elaborada pelas autoras.

A Figura 2 apresenta a comparação do período do pêndulo considerando cada um desses modelos para $L=35 \mathrm{~cm}$ e $g=9,7863$ $\mathrm{m} / \mathrm{s}^{2}$ (VALOR..., 2020). Pode-se observar que variações mais significativas entre os modelos ocorrem para ângulos maiores que $45^{\circ}$. 
A solução mais simples para o período do pêndulo simples é obtida considerando $\operatorname{sen} \theta \approx$ $\theta$, resultando na expressão

$$
T_{o}=2 \pi \sqrt{\frac{L}{g}} .
$$

Nessa aproximação, um erro menor que $5 \%$ é obtido para ângulos de até $\theta=30^{\circ}$. Como referência, o valor de $T_{\text {o }}$ para $L=35 \mathrm{~cm} \mathrm{e} g=$ $9,7863 \mathrm{~m} / \mathrm{s}^{2}$ (VALOR..., 2020) também é mostrado na Figura 2.

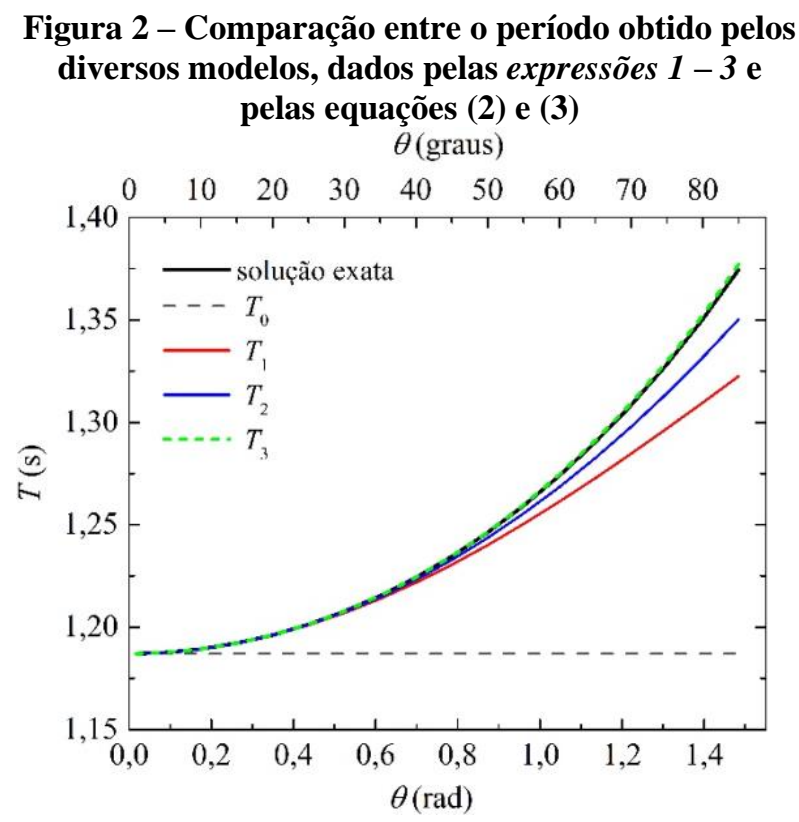

Fonte: elaborada pelas autoras.

\section{Oscilações Subamortecidas}

Para analisar o movimento de um pêndulo oscilando no ar pode-se considerar uma força viscosa $F$ que seja uma combinação de um termo linear e outro quadrático da velocidade, dada pela equação

$$
F=b \cdot v+c|v| v,
$$

onde $b$ e $c$ são constantes e $v=L \frac{d \theta}{d t}$ é a velocidade instantânea da partícula (NELSON; OLSSON, 1986; ARNOLD et al., 2011). Assim, a equação diferencial do movimento fica

$$
\frac{d^{2} \theta}{d t^{2}}+\frac{b}{m} \frac{d \theta}{d t}+\frac{c}{m}\left|\frac{d \theta}{d t}\right| \frac{d \theta}{d t}+\frac{g}{L} \operatorname{sen} \theta=0
$$

Além da equação (5) possuir somente uma solução numérica, o sinal do termo quadrático da velocidade deve ser ajustado a cada meio ciclo, como mencionado por Arnold et al.
(2011). Como a força viscosa diminui a energia mecânica do sistema e, consequentemente, a amplitude de oscilação, Nelson e Olsson (1986) obtiveram uma expressão da amplitude de oscilação em função do tempo como sendo

$$
\theta=\frac{\gamma \cdot \theta_{o} \cdot e^{-\gamma \cdot t}}{\beta \cdot \theta_{o}\left(1-e^{-\gamma \cdot t}\right)+\gamma}
$$

onde $\gamma=\frac{b}{2 m}, \beta=\frac{4}{3} \frac{\omega_{o}}{\pi} \frac{c L}{m}$ e $\omega_{o}=\sqrt{\frac{g}{L}}$.

Um pêndulo com trajetória curta e um corpo esférico de dimensões pequenas possui número de Reynolds reduzido e, como consequência, o termo da força viscosa correspondente à velocidade ao quadrado pode ser desprezado (ARNOLD et al., 2011). Nesse caso, a equação (6) se reduz a

$$
\theta=\theta_{o} e^{-\gamma \cdot t}
$$

Essa é a solução esperada para a amplitude de oscilação quando consideramos $\operatorname{sen} \theta \approx \theta \mathrm{e}$ $c \rightarrow 0$ na equação (5).

Nas próximas seções serão apresentadas: $(i)$ a metodologia de aquisição dos dados, (ii) a análise do movimento amortecido, (iii) a análise do período em função do ângulo de lançamento e (iv) a determinação da aceleração da gravidade local. Em cada uma dessas etapas serão explicitadas a metodologia e a análise de dados para o desenvolvimento das competências desejadas.

\section{EXPERIMENTO}

O arranjo experimental utilizado na aquisição de dados do pêndulo simples está ilustrado na Figura 3, que é composto por um tripé, uma haste, um transferidor e uma esfera de chumbo de 30(1) g e diâmetro 1,72(2) cm acoplada a um fio com comprimento ajustável. 
Figura 3 - Arranjo experimental para a observação do período do pêndulo simples

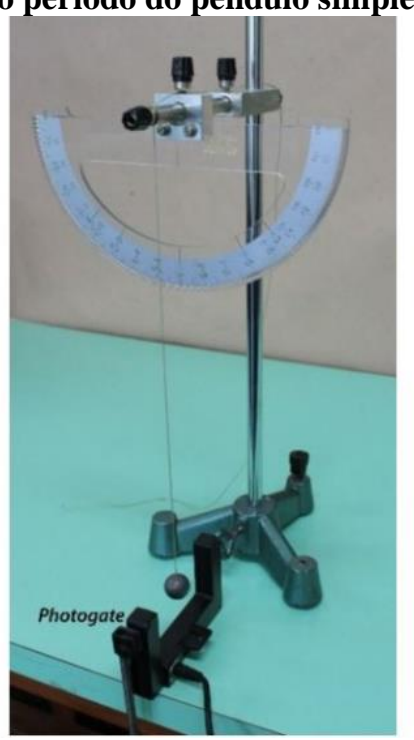

Fonte: elaborada pelas autoras.

A aquisição de dados foi feita de duas formas distintas: (i) videoanálise, com filmagem do movimento oscilatório utilizando um celular e um tripé, para posterior aquisição da posição da esfera como função do tempo através do programa livre Tracker-Video Analysis and Modeling Tool (BROWN, 2020); e (ii) com o uso de um photogate (sensor ótico) acoplado ao computador para as medidas de período usando o programa de aquisição DataStudio.

Com os dados obtidos pelo programa Tracker, além do instante de tempo $t$, também foram coletados os dados das coordenadas $x \mathrm{e}$ $y$ da esfera. Com essas coordenadas foi possível determinar a amplitude angular $(\theta(t))$ de oscilação, como mostrado na Figura 4. Os dados de período $(T)$ e amplitude angular foram obtidos como função do ângulo inicial de lançamento $\left(\theta_{o}\right)$ e do comprimento do fio $(L)$.

Com os dados obtidos pelo programa DataStudio, o período $(T)$ como função do ângulo inicial de lançamento $\left(\theta_{o}\right)$ foi obtido a partir da média das seis primeiras oscilações completas. Esse procedimento foi adotado para minimizar os efeitos da força de amortecimento que $o$ ar exerce sobre a esfera do pêndulo, conforme a análise da amplitude como função do tempo, apresentada na subseção "Amplitude como Função do Tempo".
Figura 4 - Representação esquemática do pêndulo simples para a determinação da amplitude angular $\boldsymbol{\theta}$, baseada nas coordenadas $\boldsymbol{x}$ e $\boldsymbol{y}$ da esfera de chumbo

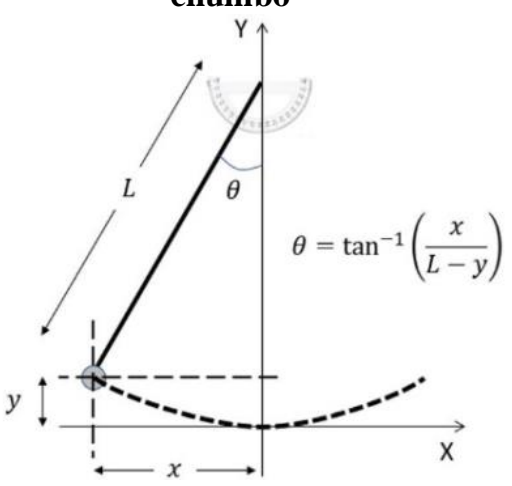

Fonte: elaborada pelas autoras.

\section{ANÁLISE EXPERIMENTAL E DISCUSSÃO}

\section{Amplitude como Função do Tempo}

As medidas da amplitude angular $(\theta)$ como função do tempo $(t)$ para o movimento do pêndulo simples mostram que, devido à presença do meio viscoso (ar), o efeito da força de amortecimento exercida sobre a esfera do pêndulo deve ser considerado na análise experimental. Nesta parte do trabalho, os conceitos envolvidos no movimento harmônico simples e força restauradora podem ser revisados junto com os estudantes para, em seguida, se discutir o movimento de um corpo oscilante em que uma força dissipativa passa a atuar no sistema, resultando em um sistema subamortecido. Os dados de $\theta$ versus $t$ podem ser analisados através de ajustes de curvas utilizando, por exemplo, o modelo de um sistema oscilatório amortecido, como o descrito pela equação (7). A partir desse desenvolvimento, é possível obter o parâmetro de amortecimento, discutir com os estudantes a qualidade do ajuste e, consequentemente, a validade do modelo escolhido, através dos parâmetros resultantes do próprio ajuste, como o coeficiente de determinação do modelo ajustado $\left(R^{2}\right)$ e gráficos de dispersão de resíduos. Com isso, o aprendizado se correlaciona com as competências relacionadas à compreensão e análise de fenômenos físicos utilizando ferramentas matemáticas, estatísticas e computacionais, validados por experimentação. 
Baseando-se no descrito acima, os dados da amplitude angular da oscilação $(\theta)$ como função do tempo $(t)$ e para diferentes ângulos de lançamento $\left(6^{\circ}-58^{\circ}\right)$, obtidos através do programa Tracker, são mostrados na Figura 5(a) para um pêndulo com $L=35 \mathrm{~cm}$. Os dados experimentais mostram claramente a diminuição de $\theta$ com o decorrer do tempo, indicando o efeito da força de atrito no movimento. Os ajustes dos dados à equação (7) foram realizados utilizando o programa livre SciDAVis (STANDISH; BENKERT; FRANKE, 2016), com o objetivo de avaliar a dependência temporal da amplitude angular com a amplitude angular inicial $\left(\theta_{0}\right)$ e o parâmetro de amortecimento $(\gamma)$.

Uma forma de avaliar a adequação do modelo ajustado é observando os gráficos de distribuição de resíduo. O resíduo é definido pela diferença entre o valor experimental e o valor ajustado. Portanto, para um modelo adequado, os resíduos são distribuídos aleatoriamente em torno de zero, apresentando valores positivos e negativos e com baixa amplitude (MASON; GUNST; HESS, 2003). Os gráficos de resíduos como função dos valores ajustados são mostrados nas Figuras 5(b - f). As análises dessas figuras mostram que o modelo de um sistema harmônico subamortecido pode ser utilizado adequadamente para ângulos de lançamento de até aproximadamente $30^{\circ}$ (Figuras $5[\mathrm{~b}-\mathrm{d}]$ ), pois os pontos do gráfico de resíduo estão distribuídos aleatoriamente em torno de $y=0 \mathrm{e}$ possuem baixa amplitude.
Figura 5 - Dados da amplitude angular como função do tempo para $L=35 \mathrm{~cm}$ e ângulos de lançamentos de $6^{\circ}, 17^{\circ}, 30^{\circ}, 49^{\circ}$ e $58^{\circ}$ (a) e seus respectivos gráficos de dispersão de resíduos resultantes dos ajustes $(\mathrm{b}-$

f). A linhas contínuas indicam o resultado
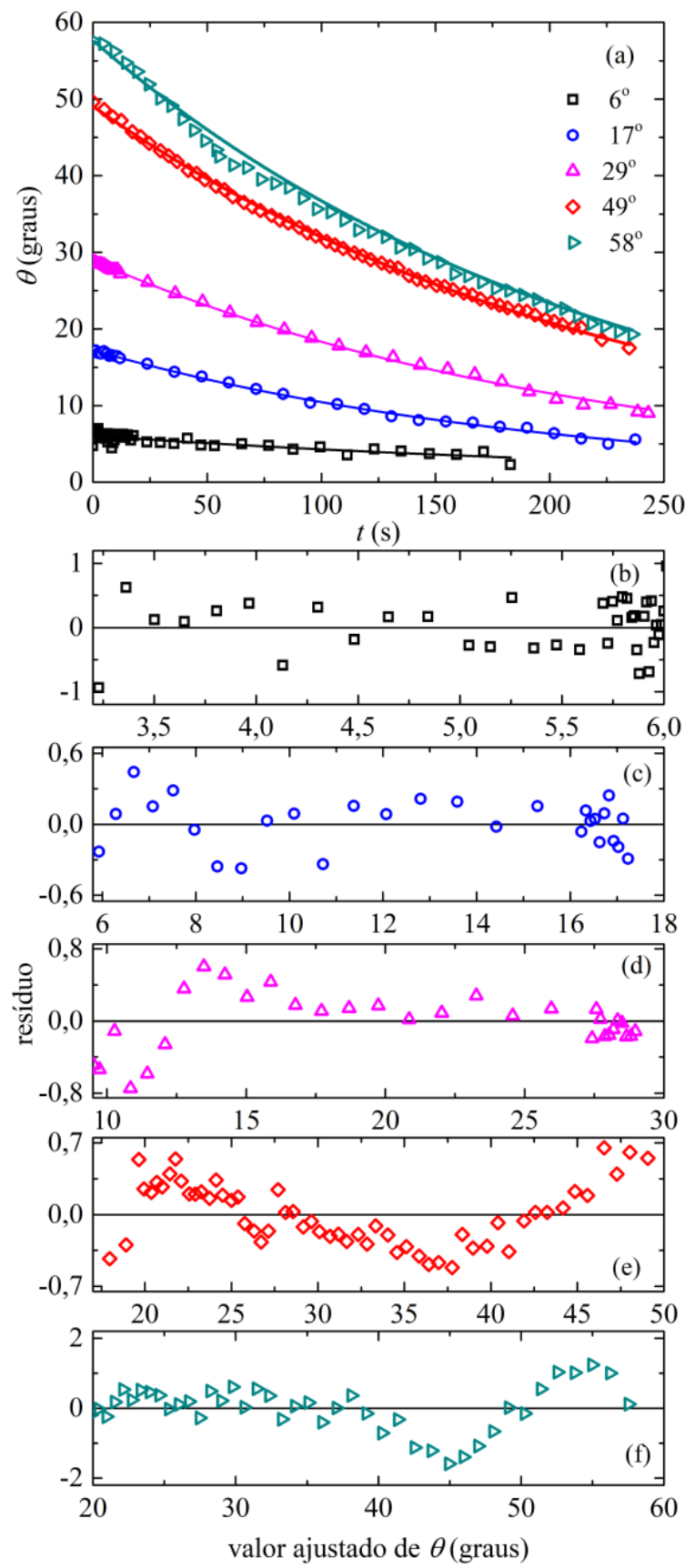

Fonte: elaborada pelas autoras.

Para ângulos de lançamento maiores, com $49^{\circ}$ e $58^{\circ}$, a aleatoriedade dos pontos no gráfico de resíduos não é mais observada, como mostram as Figuras 5(e - f), indicando que a equação (7) não descreve adequadamente o movimento do pêndulo com amplitudes angulares relativamente maiores. Portanto, a análise de resíduo pode ser usada para atestar a 
adequação do modelo utilizado (MASON; GUNST; HESS, 2003).

Os ajustes utilizando a equação (7) também foram realizados para os conjuntos de dados dos pêndulos com $L=15$ e $85 \mathrm{~cm}$. A análise conjunta dos gráficos de resíduos dos três sistemas com diferentes valores de $L$ indica que, para ângulos de lançamento maiores que $\sim 45^{\circ}$, o modelo de um sistema harmônico subamortecido, representado pela equação (7), não é adequado para descrever o movimento, pois os pontos não estão distribuídos aleatoriamente em torno de zero. A equação (7) corresponde à solução da equação (5) quando $\operatorname{sen} \theta \approx \theta$. Assim, para ângulos maiores que $30^{\circ}$, espera-se que essa equação não se ajuste de forma adequada aos dados experimentais, como de fato observado. Neste ponto, os estudantes podem discutir os limites do modelo teórico que está sendo utilizado.

O estabelecimento da adequação ou não do modelo utilizado via análise do gráfico de resíduo não necessariamente reflete a qualidade do ajuste em si, ou seja, no valor de $R^{2}$. Para ângulos de lançamento muito baixos, com $\sim 5^{\circ}$, a incerteza percentual do parâmetro ajustado de $\gamma$ é relativamente alta, maior de $5 \%$, também refletido pelo baixo valor de $R^{2}$, menor que 0,95 . Nesse caso, a relativa baixa qualidade do ajuste é devido ao método de obtenção das amplitudes angulares. Para ângulos pequenos, o erro percentual é maior, pois os valores de $x$ e $y$, mostrados na Figura 4, usados para a determinação de $\theta$, são cada vez mais reduzidos para ângulo pequenos, aumentando a incerteza na sua determinação. Portanto, para a determinação do parâmetro de amortecimento $\gamma$, foram considerados a média dos dados que se adequam ao modelo teórico proposto, avaliado a partir dos gráficos de resíduo, e os dados com boa qualidade de ajuste, avaliados pelo erro percentual menor que $2 \%$ no valor de $\gamma$. Dessa forma, os valores dos parâmetros de amortecimento foram estimados considerandose a média dos dois valores mostrados na Tabela 1, sendo $7,7 \cdot 10^{-3} ; 4,8 \cdot 10^{-3}$ e $3,9 \cdot 10^{-3} \mathrm{~s}^{-1}$ para os pêndulos com comprimentos de 15,35 e $85 \mathrm{~cm}$, respectivamente.
Tabela 1 - Resultados dos ajustes de algumas curvas mostradas na Figura 5. Os parâmetros mostrados são a amplitude angular inicial $\left(\theta_{o}\right)$ e sua incerteza percentual $\left(\sigma_{\theta_{o}}\right)$; o parâmetro de amortecimento $(\gamma)$ e sua incerteza percentual $\left(\sigma_{\gamma}\right) . R^{2}$ fornece a qualidade do ajuste

\begin{tabular}{|c|c|c|c|c|c|}
\hline 坖 & $\theta_{o}$ (graus) & $\begin{array}{l}\sigma_{\theta_{o}} \\
(\%)\end{array}$ & $\begin{array}{c}\gamma \\
\left(10^{-3} \mathrm{~s}^{-1}\right)\end{array}$ & $\begin{array}{c}\sigma_{\gamma} \\
(\%)\end{array}$ & $R^{2}$ \\
\hline \multirow{2}{*}{15} & $30,941(61)$ & 0,20 & $8,20(12)$ & 1,5 & 0,992 \\
\hline & $40,488(76)$ & 0,19 & $7,26(12)$ & 1,7 & 0,991 \\
\hline \multirow{2}{*}{35} & $17,235(71)$ & 0,41 & $4,990(62)$ & 1,2 & $\overline{0,997}$ \\
\hline & $28,970(93)$ & 0,32 & $4,570(44)$ & 1,0 & 0,998 \\
\hline \multirow{2}{*}{85} & $177,063(42)$ & 0,24 & $\overline{3,630(31)}$ & 0,85 & 0,998 \\
\hline & $30,952(84)$ & 0,27 & $4,240(37)$ & 0,86 & 0,998 \\
\hline
\end{tabular}

Fonte: elaborada pelas autoras.

Os valores de $\gamma$ variam sistematicamente com o aumento de $L$, indicando um resultado além do esperado, pois $\gamma$ é um parâmetro que depende da geometria do corpo e do coeficiente de viscosidade do meio. Nesse experimento, a mesma esfera foi utilizada em todos os ensaios e o experimento realizado no mesmo ambiente. Resultados diferentes para $\gamma$ também foram obtidos por Aggarwal, Verma e Arun (2005) para o experimento do pêndulo simples, mas com valores de $\gamma$ divergindo para ângulo de lançamento maiores que $\sim 12^{\circ}$. Os autores especulam que, para ângulos acima desse limite, a força viscosa $\left(F_{v i s}\right)$ deixaria de ser uma relação linear com a velocidade, pois $F_{v i s}$ é restrita a baixas velocidades, de forma a desprezar o efeito de turbulência.

Nesta primeira parte da análise, aplicando os conceitos de oscilações subamortecidas, foi possível verificar a validade e o limite do modelo descrito pela equação (7) com o auxílio dos gráficos de distribuição de resíduos. Para ângulos maiores que $\sim 45^{\circ}$ foi possível observar que o modelo não se ajusta adequadamente, pois o erro na aproximação $\operatorname{sen} \theta \approx \theta$ passa a ser relevante. Foi possível determinar o parâmetro de amortecimento $\gamma$ e fazer uma análise da precisão desse parâmetro, proveniente da qualidade do ajuste matemático através do parâmetro $R^{2}$. A dependência de $\gamma$ com $L$ indica uma provável necessidade de ajuste do modelo que leve em consideração uma dependência não linear com a velocidade. 


\section{Amplitude Angular como Função do Número de Oscilações}

Avaliar as aproximações matemáticas feitas para uma determinada solução de uma equação diferencial e a sua adequação aos dados experimentais também faz parte das competências a serem desenvolvidas pelos estudantes. Portanto, é importante notar que as equações (2) e (3) e as expressões 1 - 3 para o período do pêndulo simples listadas na introdução foram estabelecidas desprezando-se o efeito do amortecimento. Entretanto, esse efeito é claramente observado no movimento do sistema, como constatado na análise da subseção anterior. Dessa forma, uma alternativa para este problema é determinar o número de oscilações do pêndulo em que o valor de $g$ possa ser obtido com uma precisão escolhida pelo experimentador. Isso pode ser feito analisando a variação percentual da amplitude angular como função do número de oscilações do sistema, como mostrado na Figura 6.

Figura 6 - Variação da amplitude angular como função do número de oscilações do pêndulo simples para os pêndulos com $L=15\left(\theta_{o}=31^{\circ}\right), 35\left(\theta_{o}=\right.$ $\left.17^{\circ}\right)$ e $85\left(\theta_{o}=17^{\circ}\right) \mathrm{cm}$.

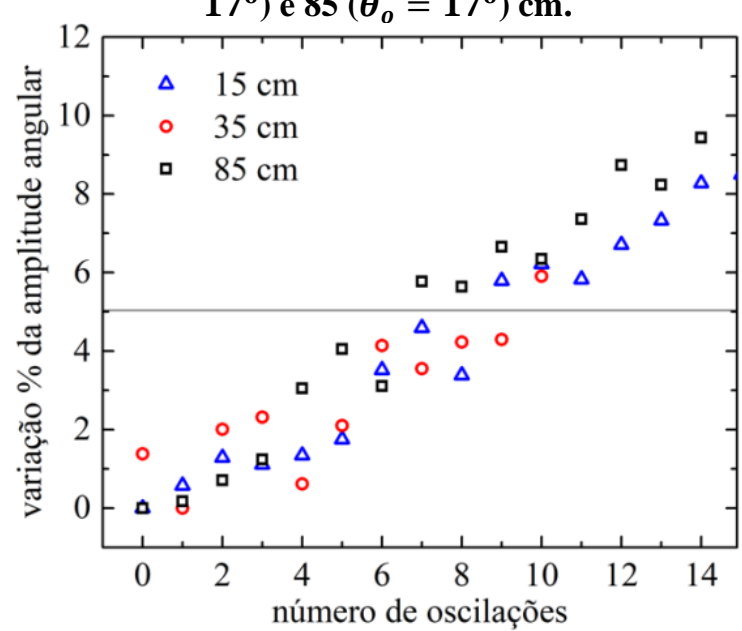

Fonte: elaborada pelas autoras.

Apesar da possibilidade de fazer essa análise independente da qualidade do ajuste ou do modelo ajustado, os dados mostrados na Figura 6 são oriundos de uma das curvas que resultaram nos melhores ajustes de cada sistema, realizado na subseção anterior. Dessa forma, são avaliados os dados com lançamento de $31^{\circ}, 17^{\circ}$ e $17^{\circ}$ para os sistemas com $L=15$, 35 e $85 \mathrm{~cm}$, respectivamente. A partir dos dados escolhidos, a perda percentual na amplitude angular como função do número de oscilações foi calculada para a escolha das condições iniciais da próxima etapa do experimento. Para esse experimento, foi estabelecido que a determinação da aceleração da gravidade local $g$ seria obtida com um erro associado ao amortecimento menor que $5 \%$.

A Figura 6 mostra que há uma variação menor que 5\% na amplitude angular para as primeiras seis oscilações. Portanto, na próxima subseção, o período do pêndulo é calculado utilizando a média dessas primeiras seis oscilações, para que os modelos apresentados na introdução sejam utilizados da mesma forma que foram apresentados, ou seja, sem a necessidade de adaptações que incluam o efeito do amortecimento no movimento do sistema. Seis oscilações para o cálculo da média do período do pêndulo é um número relativamente pequeno comparado ao comumente utilizado nos laboratórios didáticos, onde se recomenda a aquisição de 10 a 20 oscilações. Portanto, os resultados mostrados aqui revelam que o grande número de oscilações computados para a obtenção de $g$ pode não ser o melhor procedimento, dependendo do sistema. Além disso, essa análise crítica leva o estudante a refletir sobre como e em que situação a concepção de um modelo teórico pode ser validado por uma experimentação.

\section{Período como Função do Ângulo}

Para cada ângulo de lançamento, a aquisição dos períodos do pêndulo foi feita por um computador conectado a um photogate. Assim, o período médio $(\bar{T})$ como função do ângulo de lançamento foi ajustado usando os diferentes modelos, cujas expressões $(1-3)$ estão listadas na introdução. Para essas análises, os ajustes foram realizados com o auxílio do programa livre SciDAVis (STANDISH; BENKERT; FRANKE, 2016), para os ângulos de lançamento que variaram de $\theta=5$ a $60^{\circ}$ para pêndulos com $L=15,35$ e $85 \mathrm{~cm}$. Para esses conjuntos de dados, como o comprimento $(L)$ do pêndulo é conhecido, o único parâmetro de ajuste é o valor da aceleração da gravidade $(g)$. Os dados do 
pêndulo com $L=35 \mathrm{~cm}$, juntamente com as curvas ajustadas, são mostrados na Figura 7 como exemplo, e o resultado dos ajustes de todos os sistemas pode ser conferido na Tabela 2 .

Figura 7 - Período médio $(\bar{T})$ versus ângulo de lançamento $(\theta)$. As linhas tracejadas e contínuas representam as curvas ajustadas utilizando as equações (2) e (3) e expressões 1-3 listadas na Introdução

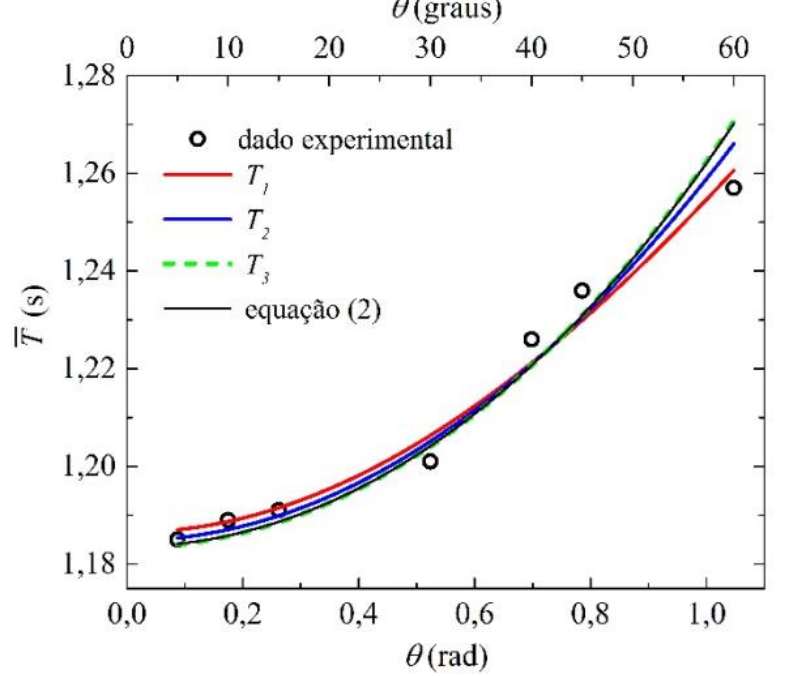

Fonte: elaborada pelas autoras.

$\mathrm{O}$ resultado do valor experimental de $g$ resultante dos ajustes das três expressões foi comparado ao valor esperado da aceleração da gravidade local $(\mathrm{g})$, cujo valor corresponde a $9,7863 \mathrm{~m} / \mathrm{s}^{2}$ (VALOR...,2020). A diferença percentual $(\varepsilon \%)$ entre os valores de $g$ experimental e esperado indica que nenhuma expressão se destaca como sendo a melhor para uma determinação mais acurada de $g$, dentro da resolução dos dados coletados. Apesar da precisão do resultado, a priori, não indica que um modelo seja o melhor, os resultados dos ajustes para os três pêndulos com $L=15,35$ e $85 \mathrm{~cm}$ analisados são extremamente consistentes entre si, sendo possível classificar as expressões utilizadas de acordo com a precisão obtida em $g$. Ao analisar a incerteza relativa $\left(\sigma_{g} / g\right)$, o modelo que melhor se ajusta aos dados experimentais é o dado pela expressão 1, apresentando incerteza relativa de 0,$34 ; 0,26$ e $0,12 \%$ para $L=15,35$ e $85 \mathrm{~cm}$, respectivamente. Em seguida, tem-se a expressão 2 e 3 . Esses resultados são consistentes com os dados observados nos gráficos de resíduo. Os gráficos de resíduo resultantes dos ajustes com a expressão 1 e 2 contêm pontos que estão distribuídos aleatoriamente em torno de $y=0$ e possuem baixa amplitude. Para a expressão 3, observa-se um gráfico de resíduo com viés para valores positivos.

Tabela 2 - A primeira coluna corresponde às expressões dos modelos listados na introdução, $g$ é o valor da aceleração da gravidade experimental e $\varepsilon \%$ é a diferença percentual entre o valor experimental e o esperado, considerando a incerteza na medida

\begin{tabular}{|c|c|c|c|c|c|c|}
\hline \multirow{2}{*}{ Expr. } & \multicolumn{2}{|c|}{$L=15 \mathrm{~cm}$} & \multicolumn{2}{c|}{$35 \mathrm{~cm}$} & \multicolumn{2}{c|}{$85 \mathrm{~cm}$} \\
\cline { 2 - 7 } & $g$ & $\varepsilon \%$ & $g$ & $\varepsilon \%$ & $g$ & $\varepsilon \%$ \\
\hline 1 & $\begin{array}{c}9,513 \\
(32)\end{array}$ & 2,5 & $\begin{array}{c}9,816 \\
(26)\end{array}$ & 0,04 & $\begin{array}{c}9,706 \\
(12)\end{array}$ & 0,70 \\
\hline 2 & $\begin{array}{c}9,541 \\
(47)\end{array}$ & 2,0 & $\begin{array}{c}9,843 \\
(32)\end{array}$ & 0,25 & $\begin{array}{c}9,739 \\
(15)\end{array}$ & 0,33 \\
\hline 3 & $\begin{array}{c}9,565 \\
(31)\end{array}$ & 1,6 & $\begin{array}{c}9,866 \\
(41)\end{array}$ & 0,39 & $\begin{array}{c}9,767 \\
(32)\end{array}$ & 0 \\
\hline
\end{tabular}

Fonte: elaborada pelas autoras.

Os resultados mais precisos e acurados de $g$ experimental foram obtidos para os pêndulos de maior comprimento, com $L=35$ e $85 \mathrm{~cm}$. O erro de $g$ experimental em relação ao valor esperado de $9,7863 \mathrm{~m} / \mathrm{s}^{2}$ foi de 1,6 a $2,5 \%$ para $L=15 \mathrm{~cm}$, de 0,04 a $0,39 \%$ para $L=35 \mathrm{~cm}$ e de 0 a $0,70 \%$ para $L=85 \mathrm{~cm}$. É interessante notar que, apesar da aquisição dos dados de período terem sido obtidos automaticamente por um sensor acoplado ao computador, os resultados indicam que maior precisão e acurácia em $g$ são obtidos para pêndulos com maiores períodos. Considerando os resultados dos ajustes das expressões 1 e 2, que foram os modelos mais adequados, o valor mais acurado de $g$ experimental foi $9,816(26) \mathrm{m} / \mathrm{s}^{2}$, com uma diferença percentual de apenas $0,04 \%$, considerando a incerteza, em relação ao valor esperado.

Caso os estudantes tenham acesso a um programa comercial de ajuste de dados ou tenham conhecimento da linguagem Python (SciPy), a solução exata, dada pela equação (2), também pode ser ajustada aos dados. Esse ajuste é mostrado na Figura 7, juntamente com outros modelos. Os resultados obtidos de $g$ foram 9,563(60); 9,864(40) e 9,764(30) $\mathrm{m} / \mathrm{s}^{2}$ para os pêndulos com $L=15,35$ e $85 \mathrm{~cm}$, respectivamente. Entretanto, observa-se um 
gráfico de resíduo com viés para valores positivos, como no resultado da expressão 3.

Neste ponto o estudante deve ser capaz de avaliar cada um dos modelos matemáticos utilizados para fazer o ajuste dos dados experimentais. A partir da análise desses modelos é possível discutir os limites da sua validade.

\section{Período como Função do Comprimento}

Nesta seção vamos considerar a equação (3) para determinar a aceleração da gravidade local $g$. Anteriormente, foi observado que o movimento do pêndulo simples é mais bem descrito por um sistema subamortecido e que, para minimizar esse efeito, seis oscilações foram consideradas para estudar a dependência do período do pêndulo com o ângulo de oscilação. Para que a equação (3) seja utilizada, o período médio $(\bar{T})$ foi obtido a partir das primeiras seis oscilações para minimizar o efeito do amortecimento e para um ângulo de lançamento de $5^{\circ}$ para que a aproximação $\operatorname{sen} \theta \approx \theta$ seja válida. Para esse ângulo, a diferença de $\operatorname{sen} \theta$ para $\theta$ é de apenas $0,13 \%$. A aquisição dos dados foi feita utilizando do programa Tracker para pêndulos com comprimentos $(L)$, variando de 20 a $90 \mathrm{~cm}$. É importante notar que, diferentemente da subseção "Amplitude como Função do Tempo" e mesmo para um ângulo pequeno, a incerteza em $T$ não depende das incertezas nas posições $x$ e $y$ da Figura 4.

Os dados do período médio ( $\bar{T}$ ) como função do comprimento $(L)$ do pêndulo podem ser convenientemente tratados através da anamorfose, ou seja, da linearização da equação (3):

$$
T^{2}=\frac{4 \pi^{2}}{g} L
$$

Assim, com o ajuste linear de $T^{2}$ versus $L$, é possível obter o valor da aceleração da gravidade $(g)$, com a determinação do coeficiente angular. Com os recursos computacionais facilmente acessíveis, é possível que a anamorfose seja um recurso desnecessário, entretanto, uma reta é muito mais simples de ser analisada do que uma curva e ainda é possível aproveitar-se de modelos estatísticos de funções lineares para a determinação e avaliação dos parâmetros. Além disso, por ser simples, uma reta representa um recurso mais didático para os alunos sem muita experiência com análise de dados.

Os dados de $T^{2}$ versus $L$ são mostrados na Figura 8 para um ângulo de lançamento $\theta_{0}=5^{\circ}$. Do resultado do ajuste linear desses dados, obtém-se um coeficiente angular igual a $4,0239(88) \mathrm{s}^{2} / \mathrm{m}$, que é igual a $4 \pi / g$, de acordo com a equação (8). Desta forma, o valor experimental obtido é $g=9,811(21) \mathrm{m} / \mathrm{s}^{2}$, que é compatível com o valor esperado de 9,7863 $\mathrm{m} / \mathrm{s}^{2} \quad$ (VALOR...,2020), considerando a incerteza. O valor de $R^{2}$ igual 0,9998 indica uma boa precisão do ajuste e o gráfico de resíduo indica que a distribuição de resíduos é aleatória e com pequena amplitude, atestando a adequação do modelo linear para os dados de $T^{2}$ versus $L$.

Figura 8-T $T^{2}$ versus $L$, para $\theta_{o}=5^{\circ}$. A linha sólida representa 0 ajuste linear.

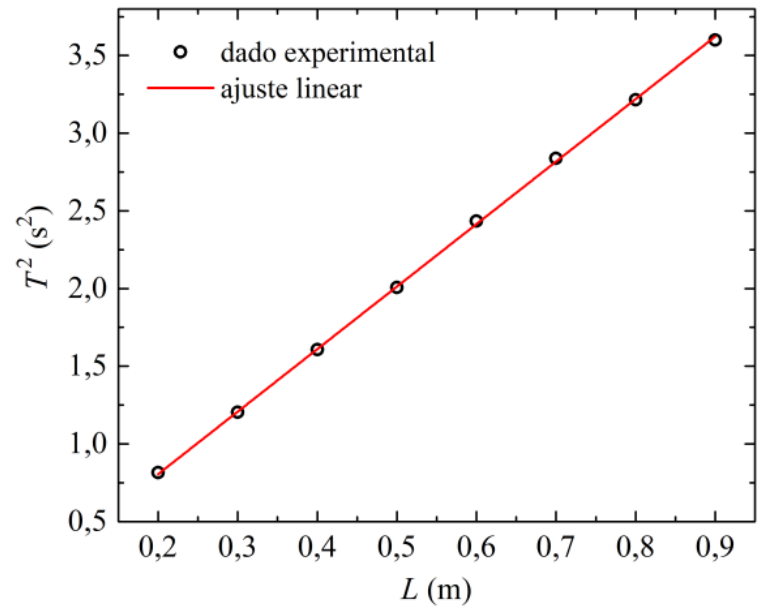

Fonte: elaborada pelas autoras.

Essa análise final mostra que é possível obter um resultado experimental com excelente acurácia e precisão, com incerteza relativa de apenas $0,22 \%$ na determinação de $g$, através da coleta criteriosa dos dados, atentando tanto para o efeito do amortecimento quanto à aproximação usada no modelo, como por exemplo $\operatorname{sen} \theta \approx \theta$. 


\section{APLICAÇÃO DA PROPOSTA EM AMBIENTE VIRTUAL}

A proposta de desenvolvimento de competências aqui apresentada foi aplicada no segundo semestre de 2020, para alunos cursando a disciplina Física 2, ministrada no segundo semestre de engenharia no período diurno. Essa disciplina é semestral, compondo os tópicos "Oscilações e Ondas" e "Termodinâmica" e foi ministrada de forma totalmente virtual. Ela inclui três encontros semanais de 100 minutos cada: dois para os tópicos de teoria e um para a realização de atividades práticas experimentais, momento em que foi aplicada a proposta aqui colocada. Essa proposta experimental foi desenvolvida ao longo de cinco semanas consecutivas.

A experiência foi dividida em três etapas, que incluíram (1) a montagem do pêndulo simples pelos alunos, utilizando os materiais que tinham disponíveis; (2) a aquisição dos dados utilizando o Tracker; e (3) a análise dos dados com o objetivo de avaliar o movimento amortecimento do pêndulo, a dependência do período com o ângulo de lançamento e a medida da aceleração gravitacional. Assim, os alunos utilizaram a expressão 2 e as equações (3) e (7) para a análise dos seus dados. Os resultados e as análises foram apresentados de forma oral e escrita.

Após a finalização da experiência, foi solicitado que os alunos respondessem a um questionário para a avaliação da percepção deles sobre as três competências associadas à $(i)$ aplicação de métodos científicos para a análise de fenômenos físicos; (ii) condução de experimentos e interpretação de resultados; e (iii) modelagem matemática de sistemas físicos (CNI, 2018; CNI, 2020). A Escala de avaliação do questionário variou de 0 (muito fraco) a 5 (excelência). Além dessa avaliação, deixamos um espaço em aberto para comentários gerais. Recebemos 127 respostas, de um total de 194 alunos, que foram compiladas e os resultados são apresentados na Tabela 3.

De acordo com os resultados obtidos, fica evidente que os alunos consideram que essas competências foram desenvolvidas por eles, mesmo em um ambiente virtual.
Tabela 3 - Resultado da percepção dos alunos sobre seu desenvolvimento das competências trabalhadas no experimento proposto

\begin{tabular}{|c|c|c|c|}
\cline { 2 - 4 } \multicolumn{1}{c|}{} & $($ i $)$ & $($ ii $)$ & (iii) \\
\hline $0-1$ & $1,6 \%$ & $1,6 \%$ & $1,6 \%$ \\
\hline $2-3$ & $5.5 \%$ & $9.4 \%$ & $9.4 \%$ \\
\hline $4-5$ & $92.9 \%$ & $89.0 \%$ & $89.0 \%$ \\
\hline
\end{tabular}

Fonte: elaborada pelas autoras.

Em relação aos comentários gerais, vale destacar os citados abaixo, que demonstram a percepção dos alunos sobre as competências trabalhadas: (a) "Foi um trabalho bem extenso $e$ de certa forma desafiador, foi incrivel trabalhar e experimentar sobre o assunto..."; (b) "Aprendi melhor sobre os cuidados que se deve ter para realizar experiências de Física. Foi uma ótima experiência entender melhor sobre o mhs e mha na prática."; e (c) "Foi um projeto que me auxiliou a compreender melhor a matéria de teoria, uma vez que a prática sempre é a melhor maneira de se aprender algo.".

\section{CONSIDERAÇÕES FINAIS}

Este trabalho apresentou detalhes do procedimento experimental e análise de dados do experimento do pêndulo simples com o objetivo de desenvolver competências associadas à utilização de metodologia científica e à tomada de decisão para a condução de experimentos, além de possibilitar, aos estudantes, a compreensão dos limites de validade da modelagem matemática de dados experimentais. Por ser um experimento largamente utilizado em cursos de graduação na área de exatas e de fácil ajuste dos parâmetros, foi possível explorar procedimentos experimentais mais cuidadosos de aquisição dos dados, assim como a utilização de modelos matemáticos, com análise crítica através de critérios estatísticos. Dessa forma, esperamos que o aluno desenvolva um o pensamento crítico, que o conduza a uma reflexão sobre o valor da aceleração da gravidade obtido, avaliando a necessidade de eventuais correções e considerando as limitações dos modelos teóricos utilizados. 


\section{Agradecimentos}

As autoras gostariam de agradecer aos técnicos ao Centro Universitário FEI pelo apoio logístico e ao Prof. Dr. Roberto B. B. Santos pelas sugestões.

\section{REFERENCIAS}

ABENGE. Diretrizes para o Curso de Engenharia - ABENGE, MEI/CNI, Brasília, jan. 2018. Disponível em: < http://www.abenge.org.br/documentos/Propos taDCNABENGEMEI_CNI.pdf $>$. Acesso em: 11 set. 2020

AGGARWAL, N.; VERMA, N.; ARUN, P. Simple pendulum revisited. European Journal of Physics, v. 26, n. 3, p. 517, 2005.

ARNOLD, F. J. et al. Estudo do amortecimento do pêndulo simples: uma proposta para aplicação em laboratório de ensino. Revista Brasileira de Ensino de Física, v. 33, n. 4, p. 4311-4311, 2011.

BROWN, Douglas. Tracker video analysis and modeling tool, 2020.

CNI. Recomendações para o Fortalecimento e Modernização do Ensino de Engenharia no Brasil, Brasília, mar. 2018. Disponível em: https://www.portaldaindustria.com.br/publica coes/2018/3/destaques-de-inovacaorecomendacoes-para-o-fortalecimento-emodernizacao-do-ensino-de-engenharia-nobrasil/. Acesso em: 11 set. 2020.

CNI. Documento de Apoio à Implantação das DCNs do Curso de Graduação em Engenharia, Brasília: CNI, 2020. Disponível em:

https://www.portaldaindustria.com.br/publica coes/2020/6/documento-de-apoio-

implantacao-das-dens-do-curso-de-graduacaoem-engenharia/. Acesso em: 11 set. 2020.

FULCHER, L. P.; DAVIS, B. F. Theoretical and experimental study of the motion of the simple pendulum. American Journal of Physics, v. 44, n. 1, p. 51-55, 1976.
LIMA, F. M. S.; ARUN, P. An accurate formula for the period of a simple pendulum oscillating beyond the small angle regime. American Journal of Physics, v. 74, n. 10, p. 892-895, 2006.

MARION, J. B.; THORNTON, S. T. Classical Dynamics of Particles and Systems. 3. ed. Philadelphia: Saunders, 1988.

MASON, Robert L.; GUNST, Richard F.; HESS, James L. Statistical design and analysis of experiments: with applications to engineering and science. 2. ed. Nova Jersey: John Wiley \& Sons, 2003.

MEC. Diretrizes Curriculares Nacionais do Curso de Graduação em Engenharia, Brasília, 23 jan. 2019. Disponível em: http://portal.mec.gov.br/index.php?option=co $\mathrm{m}$ docman\&view $=$ download\&alias $=109871$ pces001-19-1\&category_slug=marco-2019pdf\&Itemid=30192. Acesso em: 11 set. 2020.

NELSON, Robert A.; OLSSON, M. G. The pendulum-Rich physics from a simple system. American Journal of Physics, v. 54, n. 2, p. 112-121, 1986.

RUGARCIA, Armando et al. The future of engineering education I. A vision for a new century. Chemical Engineering Education, v. 34 , n. 1 , p. 16-25, 2000.

SMITH, C. J. A Degree Physics: Part I. The General Properties of Matter. 2 ed. Londres: Edward Arnold, 1960).

STANDISH, R.; BENKERT, T.; FRANKE, K. SciDAVis. Free Application for Scientific Data Analysis and Visualization, 2016.

VALOR DA ACELERAÇÃO DA GRAVIDADE LOCAL. Pode ser obtido pelos sites WolframAlpha e SensorsOne. Disponíveis em: $<$ https://www.wolframalpha.com/widgets/vie w.jsp?id=e856809e0d522d3153e2e7e8ec263b f2> e <https://www.sensorsone.com/localgravity-calculator/>. A localização da latitude e elevação local podem ser obtidas no site Geoplaner. Disponível em: <https://www.geoplaner.com/>. Acesso em: 11 set. 2020. 
WANKAT, P. C.; BULLARD, L. G. The future of engineering education-Revisited. Chemical Engineering Education, v. 50, n. 1, p. 19-28, 2016.
WORLD ECONOMIC FORUM. The future of jobs report 2018. Disponível em: http://www3.weforum.org/docs/WEF_Future_ of_Jobs_2018.pdf>. Acesso em: 11 set. 2020.

\section{DADOS BIOGRÁFICOS DOS AUTORES}
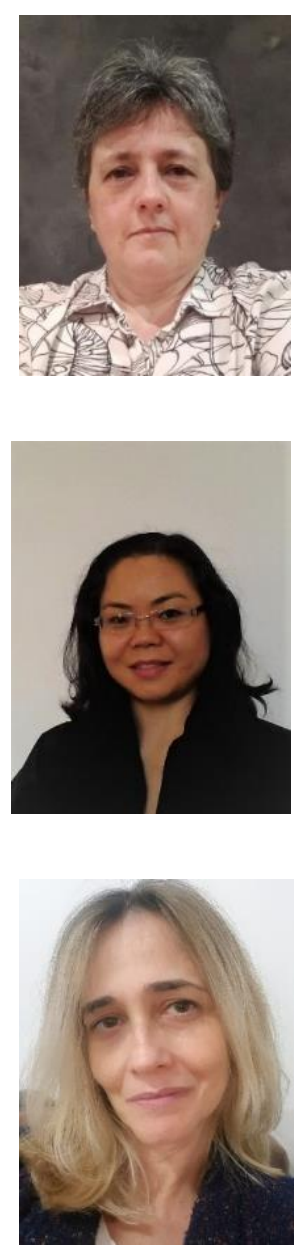

Eliane F. Chinaglia - Bacharelado em Física (USP, 1994), Mestrado em Ciências (ISP, 1997), Doutorado em Ciências (USP, 2002) e Pós-doutorado em Engenharia de Materiais (EPUSP, 2003). Atua como professora do Centro Universitário FEI desde 2006 pelo departamento de Física, onde coordenou as disciplinas Física 1, 2 e Física Moderna. Orienta alunos de Iniciação Científica e Didática e atua nas áreas de Ensino de Física para Engenharia, Fabricação e Caracterização de Filmes Finos Metálicos e Óxidos e caracterização de dispositivos a base de grafeno.

Sueli Hatsumi Masunaga - Graduada em Física pela USP (2002), com mestrado (2005) e doutorado (2009) em Ciências, programa Física, pela USP e Pós-doutorado (2010-2016) na área de Física da Matéria Condensada pela USP e Montana State University. Atua como professora no Centro Universitário FEI desde 2017 pelo departamento de Física e como orientadora de alunos de Iniciação Científica, sendo que as áreas de atuação e interesse são ensino de física e pesquisa de materiais nanoestruturados, monocristalinos e policristalinos, envolvendo caracterização estrutural, magnética, térmica e de transporte eletrônico.

Simone Camargo Trippe - Possui bacharelado em Física pela USP (1996), mestrado em Física pela USP (1999) e doutorado em Engenharia Elétrica pela USP (2003). Desde 2011 é professora adjunta II do Centro Universitário da FEI em São Bernardo do Campo. Tem experiência na área de Engenharia Elétrica, com ênfase em processos de microeletrônica, nanoeletrônica e desenvolvimento de dispositivos por litografia utilizando escrita direta com feixe de elétrons e técnicas correlatas. Atua principalmente nos seguintes temas: caracterização, propriedades elétricas, deposição e aplicação elétrica, nanolitografia por feixe de elétrons e microscopia eletrônica. Atualmente desenvolve pesquisa na área de educação e em caracterização de materiais, especialmente a caracterização e produção de grafeno e óxido de grafeno. 